\title{
MRSA DIVERSITY AND THE EMERGENCE OF LA-MRSA IN A LARGE TEACHING HOSPITAL IN SLOVENIA
}

\author{
BoŽEnA KotNIK KEVORKIJAN ${ }^{1}$, ŽIVA PETROVIČ ${ }^{2}$, ALEKSANDER KOCUVAN ${ }^{3}$ and \\ MAJA RUPNIK ${ }^{2,3 *}$ \\ ${ }^{1}$ Department of Infectious Diseases, University Clinical Centre Maribor, Maribor, Slovenia \\ ${ }^{2}$ Centre for Medical Microbiology, National Laboratory for Health, Environment and \\ Food, Maribor, Slovenia \\ ${ }^{3}$ Faculty of Medicine, University Maribor, Maribor, Slovenia
}

(Received: 25 September 2018; accepted: 16 November 2018)

\begin{abstract}
The methicillin-resistant Staphylococcus aureus (MRSA) is one of the major causes of a variety of infections in hospitals and the community. One of the most prominent changes in the MRSA epidemiology is the emergence of livestockassociated MRSA (LA-MRSA) strains in the human population. The aim of this study was to follow the MRSA epidemiology in a large teaching hospital during an 8-year time period (2006-2013). Altogether 519 MRSA, cultured from screening or clinical samples, were distributed into 77 spa types, of which three (t003 and t001, associated with $\mathrm{CC} 5$; and $\mathrm{t} 015$; associated with CC45) were the most common. LA-MRSA-associated spa types (t011, t034, t108, t899; associated with CC398) started to emerge in the year 2009 and continued to be found annually at a frequency from $3.9 \%$ to $12.7 \%$ of all MRSA strains examined. Only 6 of 27 LA-MRSA strains were associated with infections.
\end{abstract}

Keywords: spa typing, LA-MRSA, Staphylococcus aureus, animals, colonization

\section{Introduction}

The methicillin-resistant Staphylococcus aureus (MRSA) is one of the most important multidrug resistant microorganisms and primarily causes healthcareassociated (HA-MRSA) infections [1, 2]. During the past two decades, MRSA was also increasingly reported in the community and this type was designated a commmunity-associated MRSA (CA-MRSA). The CA-MRSA and HA-MRSA could be differentiated epidemiologically by association with nosocomial settings,

*Corresponding author; E-mail: maja.rupnik@nlzoh.si 
as well as the time of onset in the hospital. They could also be differentiated pheno- and genotypically by attribution to clonal lineages (complexes), the type of SCCmec element, antibiotic resistance patterns, and the virulence potential $[3,4]$. However, the distinction between both groups is disappearing [3]. The CA-MRSA is acquiring additional antibiotic resistances and both types are currently circulating in hospitals as well as in the community. Some successful clones have spread worldwide [5-7]. Since 2005, a group of MRSA associated with farm animals, termed as livestock-associated MRSA (LA-MRSA), started to emerge in the human population $[8,9]$. The most common LA-MRSA group is ST398 with a large number of different spa types [7, 8, 10, 11]. Although LA-MRSA represents less than $10 \%$ of strains isolated from humans in the majority of countries, it is important to follow the national and local rates in order to adapt patient screening if necessary [11].

In Slovenia, the MRSA is well controlled and the MRSA colonization rate is used as a quality indicator $[12,13]$. The hospitals have issued guidelines for the prevention of MRSA healthcare-associated transmission, including hand hygiene, which is the most important infection control measure, patient isolation, and surveillance of patients at risk [14]. However, despite good hospital controls, at present, a detailed MRSA epidemiology in Slovenia is less than thoroughly investigated, as the focus is mainly on the CA-MRSA [15-17]. Newly described $m e c \mathrm{C}$-positive isolates have been found in a large collection of 395 CA-MRSA, isolated nationwide from 2006 to 2013 [18]. In the same collection, a subset of MRSA isolated in 2010 were spa- and MLST-typed, and tested for presence of toxin genes [19]. Of 151 isolates, 9.9\% belonged to ST398, representing the first report of LA-MRSA strains in Slovenia. Slovenian laboratories also participated in large international studies on $S$. aureus bloodstream infections [1, 20], MRSA in intensive care units (ICUs) [21], and an ECDC cross-sectional study on LAMRSA for the year 2013 [11].

In this context, the aim of this study was to analyze the epidemiology of MRSA spa types in a single large teaching hospital during an extended (8-year) time interval, and to determine the possible presence and proportion of LA-MRSA.

\section{Materials and Methods}

\section{Hospital setting}

The University Clinical Centre Maribor (UCCM) is a tertiary teaching hospital, located in the North-East region of Slovenia, serving a population of 
400,000. It has 1,300 beds with ca. 55,000 discharges (364,000 patient days) per year including the study period. The hospital had an active MRSA surveillance since 1999 and patients with at least one of the following risk factors are included in the screening: previous hospitalization within the last 12 months, transfer from another hospital or from a long-term care facility, previously known colonization with MRSA, patients with chronic wounds, all patient in critical care units, patients undergoing clean elective and implant surgery, patients on peritoneal dialysis, patients with more than $24 \mathrm{~h}$ room contact with a patient who was a confirmed as MRSA carrier, and healthcare workers at the wards with proven MRSA cases at the time of an epidemic.

\section{Ethics}

Ethical approval for this study was obtained from the National Medical Ethic Committee (no. KME84/08/12).

\section{Isolation and characterization of MRSA}

For the strain selection, isolation, and characterization, we followed the methods of Kotnik-Kevorkijan et al. [22]. S. aureus cultivated from clinically relevant samples were recognized as MRSA because of the resistance to cefoxitin, and were subsequently confirmed with mecA testing. For surveillance samples, the conventional culture media, including MRSA-screening plates $\left(\mathrm{CHROMID}^{\circledR}\right.$ MRSA, bioMerieux, Marcy-l'Etoile, France) and trypticase soy broth containing $\mathrm{NaCl}$, were used. MRSA strains were confirmed by PCR amplification of the mecA gene by a modification of previously published methods [23, 24]. An in-house method is established and is based on amplification of three different PCR products; two are $S$. aureus-specific and one targets mecA gene. The mec $\mathrm{C}$ testing was introduced in the second half of the year 2013 for strains that were resistant to cefoxitine and negative for mecA. No strains fulfilling these criteria were detected within the study interval. All isolates were frozen at $-70{ }^{\circ} \mathrm{C}$ until further characterization. Only the first isolate of each patient was stored.

\section{Antimicrobial susceptibility testing}

Susceptibility testing, using a standard selection of antibiotics for S. aureus, was performed by disk diffusion according to the CLSI Performance Standards for Antimicrobial Susceptibility Testing (CLSI document M100; 
https:/clsi.org/standards/products/microbiology/documents/m100/). The antibiotics tested were tetracycline, clindamycin, erythromycin, ciprofloxacin, gentamycin, tobramycin, mupirocin, linezolid, trimethoprim-sulfamethoxazole, teicoplanin, vancomycin, and netilmicin. Since 2011, the susceptibility to vancomycin has been assessed by E-test.

\section{Strain selection and spa typing}

The MRSA, which were typed, were isolated between January 2006 and December 2013 from either surveillance or clinical samples. Only the first isolate of each patient was included in the analysis.

The isolates were thawed and cultured on blood agar. After DNA isolation using the QIAamp DNA Kit (QIAgen, Venlo, Netherlands), the spa region was amplified using primers and conditions as described previously [25] and sequenced by a commercial service (MWG, Martinsried, Germany). The spa types were assigned using the Ridom StaphType software (Ridom GmbH, Würzburg, Germany) [25]. The Based Upon Repeat Pattern (BURP) algorithm of Ridom StaphType software was used to analyze the similarity of spa types and the grouping into clusters using the conditions described in the study of Mellmann et al. [26].

\section{Results}

Overall, 519 MRSA isolates from UCCM were spa typed between the years 2006 and 2013. The cultures were mostly obtained from surveillance swabs (nose, throat, skin, or wound) or, in rare cases, from other surveillance samples (urine or feces) and from patients with developed infections (blood culture, urine sample, swabs from wound, eye, ear, and oral mucosa). Altogether 77 spa types were found (Table I), three of which (t003, t001, and t015) were represented by more than 50 isolates each. Three further spa types ( $\mathrm{t} 041$, t091, and t728) were also frequently isolated during this time period, whereas 40 spa types were represented only by a single isolate (2 LA-MRSA and 38 non-LA-MRSA spa types; Table I). Using the BURP algorithm, three large and several small clusters were obtained (Figure 1). Twelve spa types were singletons ( $\mathrm{t} 151, \mathrm{t} 316, \mathrm{t} 334, \mathrm{t} 791, \mathrm{t} 899, \mathrm{t} 1048, \mathrm{t} 1179, \mathrm{t} 1321, \mathrm{t} 3824, \mathrm{t} 4230$, t4365, and t14168) and additional six were excluded from the analysis due to the low number of repeats (t026, t288, t390, t458, t808, and t1040).

The spa types associated with the LA-MRSA (t011, t034, t108, and t899) started to emerge in the year 2009 (Table I). These types represented 5.2\% 
Table I. Overview of spa types detected in a single hospital in 8-year period

\begin{tabular}{|c|c|c|c|c|c|c|c|c|c|c|}
\hline spa type & $\mathrm{CC}^{\mathrm{a}}$ & 2006 & 2007 & 2008 & 2009 & 2010 & 2011 & 2012 & 2013 & $\Sigma$ \\
\hline t003 & CC5 & 6 & 8 & 6 & 28 & 16 & 19 & 44 & 24 & 151 \\
\hline t001 & CC5 & 38 & 20 & 6 & 4 & 1 & - & - & - & 69 \\
\hline t015 & $\mathrm{CC} 45$ & - & 2 & 2 & 12 & 15 & 8 & 7 & 6 & 52 \\
\hline t041 & CC5 & 2 & - & 12 & 6 & 2 & 3 & 1 & - & 26 \\
\hline t091 & $\mathrm{CC} 7$ & - & 11 & 1 & 2 & 3 & - & 1 & 1 & 19 \\
\hline $\mathrm{t} 728$ & nd & - & 2 & - & 3 & 6 & 2 & 2 & 4 & 19 \\
\hline t011 & CC398 & - & - & - & 3 & 6 & 1 & 1 & 6 & 17 \\
\hline t034 & CC398 & - & - & - & 2 & - & 1 & 2 & 3 & 8 \\
\hline $\mathrm{t} 108$ & CC398 & - & - & - & - & 1 & - & - & - & 1 \\
\hline t899 & CC398 & - & - & - & - & - & 1 & - & - & 1 \\
\hline t002 & CC5 & - & 2 & - & 3 & 2 & 1 & - & 2 & 10 \\
\hline t005 & $\mathrm{CC} 22$ & 1 & 2 & - & 1 & 3 & - & 2 & 1 & 10 \\
\hline $\mathrm{t} 288$ & nd & 8 & 1 & - & - & - & - & - & - & 9 \\
\hline t006 & nd & - & 1 & 1 & 3 & 1 & - & - & - & 6 \\
\hline $\mathrm{t} 355$ & CC152 & 1 & - & - & 1 & 2 & 1 & - & 1 & 6 \\
\hline t008 & CC8 & - & - & - & 1 & 1 & 2 & 1 & - & 5 \\
\hline t026 & nd & - & - & - & 1 & 1 & - & 2 & 1 & 5 \\
\hline $\mathrm{t} 127$ & $\mathrm{CC} 1$ & - & - & - & 1 & 2 & 2 & - & - & 5 \\
\hline $\mathrm{t} 331$ & nd & - & - & - & - & - & 2 & 2 & 1 & 5 \\
\hline t808 & nd & - & - & - & - & - & 2 & 1 & 2 & 5 \\
\hline t5605 & nd & - & - & - & - & - & 4 & - & 1 & 5 \\
\hline t201 & nd & 1 & 1 & - & 2 & - & - & - & - & 4 \\
\hline $\mathrm{t} 3255$ & nd & - & 4 & - & - & - & - & - & - & 4 \\
\hline t3824 & nd & - & 2 & - & - & 1 & - & - & 1 & 4 \\
\hline t030 & ST239 & 3 & - & - & - & - & - & - & - & 3 \\
\hline t032 & $\mathrm{CC} 22$ & - & 3 & - & - & - & - & - & - & 3 \\
\hline t037 & ST239 & - & - & 2 & - & - & - & 1 & - & 3 \\
\hline t116 & CC45 & - & - & - & - & - & 1 & 2 & - & 3 \\
\hline t919 & nd & - & 1 & - & 1 & - & 1 & - & - & 3 \\
\hline 10 types $^{\mathrm{b}}$ & & 1 & 1 & 2 & 2 & 1 & 3 & 2 & 8 & 20 \\
\hline 38 types $^{\mathrm{b}}$ & & 3 & 6 & 1 & 4 & 4 & 6 & 5 & 9 & 38 \\
\hline $\begin{array}{l}\text { No. of typed } \\
\text { MRSA } \\
\text { strains }\end{array}$ & & 64 & 67 & 33 & 80 & 68 & 60 & 76 & 71 & 519 \\
\hline LA-MRSA & & 0 & 0 & 0 & 5 & 7 & 3 & 3 & 9 & 27 \\
\hline
\end{tabular}

Note: spa types are grouped into most prevalent types, LA-MRSA types, types with up to three isolates, and sporadic types (one or two isolates).

${ }^{\mathrm{a}} \mathrm{CC}$ deduced from spa type (based on data at Ridom server and Ref. [27]).

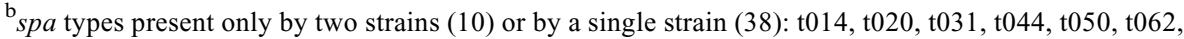
t073, t102, t122, t133, t151, t310, t316, t334, t359, t360, t390, t449, t458, t542, t548, t550, t595, t685, t688, t709, t791, t830, t950, t1040, t1048, t1179, t1321, t2986, t3432, t3445, t4072, t4230, t4272, $\mathrm{t} 4365, \mathrm{t} 5047, \mathrm{t} 5933, \mathrm{t} 7736, \mathrm{t} 8014, \mathrm{t} 10458, \mathrm{t} 10459, \mathrm{t} 14167$, and $\mathrm{t} 14168$. 


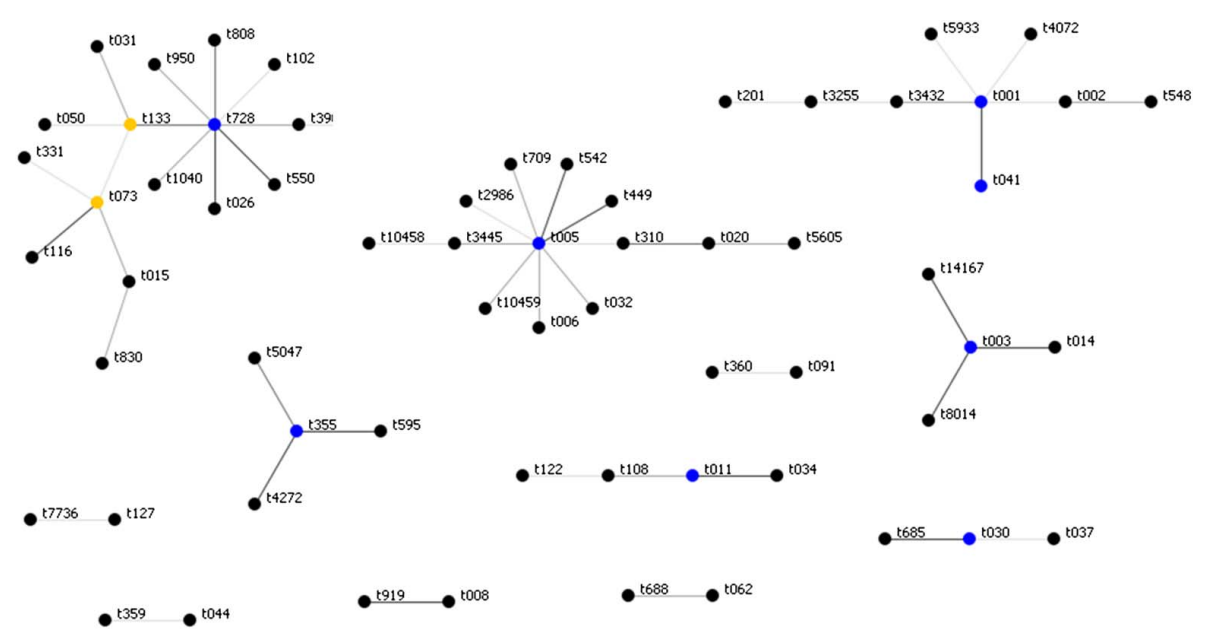

Figure 1. BURP clustering of spa types found in the hospital. The spa type with the highest founder-score is defined as the founder of the cluster (blue color); several spa types could have identically high founder-scores. The spa types with the second highest founder-score (subfounders) are marked with yellow

(27/591) of all characterized strains, and ranged from 3.9\% to $12.7 \%$ of all isolates within an individual year. Only six of 27 LA-MRSA strains were associated with infections (Table II).

Although antibiotic resistance was routinely tested during the MRSA isolation, data are shown only for the LA-MRSA (Table III). All strains were resistant to tetracycline alone or together with other antibiotics. However, all strains were susceptible to mupirocin, linezolid, trimethoprim-sulfamethoxazole, teicoplanin, vancomycin, and netilmicin.

Table II. Summary of clinically relevant LA-MRSA strains showing the patient age and gender and of clinical presentations

\begin{tabular}{lcccc}
\hline Gender & $\begin{array}{c}\text { Age } \\
\text { (years) }\end{array}$ & $\begin{array}{c}\text { Clinical diagnosis related } \\
\text { to LA-MRSA }\end{array}$ & $\begin{array}{c}\text { MRSA isolation } \\
\text { year }\end{array}$ & spa type \\
\hline Female & 44 & Pneumonia & 2010 & $\mathrm{t} 108$ \\
Female & 53 & Otitis media & 2011 & $\mathrm{t} 011$ \\
Male & 52 & Osteitis & 2012 & $\mathrm{t} 011$ \\
Male & 53 & Pneumonia & 2012 & $\mathrm{t} 034$ \\
Female & 76 & Conjunctivitis & 2013 & $\mathrm{t} 011$ \\
Female & 71 & Stomatitis & 2013 & $\mathrm{t} 011$ \\
\hline
\end{tabular}

Note: MRSA: methicillin-resistant Staphylococcus aureus; LA-MRSA: livestock-associated MRSA. 
Table III. Antibiotic resistances in strains belonging to LA-MRSA-associated spa types isolated from surveillance or clinical samples (indicated by "infection") in a single hospital from the year 2009 to 2013

\begin{tabular}{lcc}
\hline Resistance & $\begin{array}{c}\text { spa types (number of strains } \\
\text { from surveillance samples) }\end{array}$ & $\begin{array}{c}\text { spa types (number of strains } \\
\text { from clinical samples) }\end{array}$ \\
\hline $\begin{array}{l}\text { Tetracycline } \\
\text { Tetracycline and chloramphenicol }\end{array}$ & t011 (7) and t034 (2) & t011 (2) \\
$\begin{array}{l}\text { Tetracycline and ciprofloxacin } \\
\text { Tetracycline, clindamycin, and }\end{array}$ & t011 (1) & t011 (1) and t108 (1) \\
erythromycin & t011 (2) and t034 (4) & t899 (1) \\
Tetracycline, clindamycin, & - & t034 (1) \\
erythromycin, and ciprofloxacin & & - \\
Tetracycline, clindamycin, \\
erythromycin, gentamycin, and \\
tobramycin
\end{tabular}

Note: LA-MRSA: livestock-associated MRSA.

\section{Discussion}

The reported rates for UCCM between 1998 and 2007 ranged from 53 to 114 patients colonized or infected with MRSA, and MRSA represented from $4.5 \%$ to $10 \%$ of all S. aureus isolates [22]. Information for the year 2008 is not available, but from 2009 to 2013, the number of MRSA patients and the percentage of MRSA among S. aureus isolates are comparable to the previous study (Table IV).

The spa types found in this study are associated with all main globally distributed clonal complexes (CC5, CC8, CC22, and CC45) except CC30 (Table I) [27]. Some spa types are associated with the clonal complex CC152, reported from West European countries and previously suggested to be present in the Balkan region [35]. Two spa types, associated with ST239, were also found in Asia $[35,36]$. CC398 was continuously present since the year 2009. The three globally most widespread spa types (t002, t008, and t037) [28] were found, but in low proportions.

Table IV. Proportion of patients infected or colonized with MRSA during 5 out of 8 years of the study interval

\begin{tabular}{lccccc}
\hline & 2009 & 2010 & 2011 & 2012 & 2013 \\
\hline All patients with MRSA & 95 & 86 & 72 & 94 & 96 \\
Infections with MRSA & $7(7.4 \%)$ & $4(4.6 \%)$ & $1(1.4 \%)$ & $12(12.8 \%)$ & $5(5.2 \%)$ \\
All patients with $S$. aureus & 1,121 & 1,072 & 1,123 & 1,070 & 1,062 \\
Percentage of MRSA among S. aureus & 8.4 & 8.2 & 6.4 & 8.7 & 9.0 \\
Number of days in bed & 390,366 & 385,207 & 381,832 & 353,628 & 351,719 \\
Number of discharges & 61,218 & 62,689 & 60,535 & 52,497 & 56,752 \\
\hline
\end{tabular}

Note: MRSA: methicillin-resistant Staphylococcus aureus. 
Some of the most common spa types found (e.g., t001, t003, and t041) are frequently detected in several countries in Europe and worldwide. Type t001, which was predominant at the beginning of the study period, was previously reported for single Slovenian strain, isolated in 1999, and was also found in strains isolated from Poland and Germany during the same time period [29]. Similar to this study, t001 and t041 were among the predominant types reported from hospitals in Croatia, Bosnia and Herzegovina, and Serbia [30, 31]. Types t001, t041, and t003 were also commonly found in ICUs during the MOSAR study (2008-2011) in Slovenia, as well as in Italy, Greece, and Luxemburg [21].

Type t003, which replaced t001 at UCCM over time (Table I), is the second most common spa type in the Ridom server database (http://spa.ridom.de/ frequencies.shtml). This spa type was also common in clinical and surveillance MRSA strains in one of the Canadian provinces [6], the second most common MRSA type found in the second European study on $S$. aureus bloodstream infections [20], and the most commonly isolated MRSA from cases of bacteremia in a large German federal state [32]. In Germany, type t003 also represented $64.3 \%$ of all MRSA strains in residents of long-term care facilities [33]. The high proportion of t003 strains at UCCM also appeared to have at least some relation to the hospitalization of residents from nursing homes for the elderly (data not shown).

Of the two other frequently isolated spa types, t015 was also very common in a previous national study, while t728 was limited only to UCCM [19].

Reports of LA-MRSA incidence vary considerably, depending on the country and patient population. For example, a European surveillance in 2007 reported a LA-MRSA frequency ranging from $0 \%$ to $4.7 \%$, with the exception of the Netherlands (with $11.9 \%$ in the national reference laboratory and $25 \%$ in a single local laboratory) [8]. Ireland has reported low LA-MRSA levels $(0.05 \%)$ during a time period of 2010-2014 [34]. Characterization of more than 2500 MRSA isolates from an Austrian region close to UCCM found CC398 strains represented $11.11 \%$ of isolates in 2007 and $8.17 \%$ in 2012 [35]. A study across 27 European countries has identified an overall proportion of $3.9 \%$ of LA-MRSA, but in five countries (including Slovenia), the proportion was higher than $10 \%$ [11]. The proportion of $3.9 \%-12.7 \%$ of LA-MRSA isolates within an individual year correlates well with national data reported for presumptive CA-MRSA analyzed in 2010 (9.9\%; [19]).

As observed by others [21, 36, 37], the LA-MRSA was mostly isolated from surveillance cultures and rarely caused disease in the studied hospital.

Pigs and cattle are considered the main reservoir for the LA-MRSA, and people having professional contact with farm animals or living at industrial farms are considered to have increased risk for colonization [9]. As the study was carried out retrospectively, no further epidemiological data on possible animal contact are 
available, but a high proportion of LA-MRSA is consistent with the location of UCCM in the more agriculturally oriented North-East region of Slovenia.

In summary, we found a broad diversity among MRSA strains in UCCM, with the predominance of spa types associated with CC5 and CC45. LA-MRSA represented up to $12.7 \%$ of all MRSA and started to emerge in the year 2009 .

\section{Acknowledgements}

This work was supported by University Clinical Centre Maribor (grant IRP-2013/02-04 to BKK).

\section{Conflict of Interest}

The authors report no conflict of interest.

\section{References}

1. European Centre for Disease Prevention and Control: Antimicrobial Resistance Surveillance in Europe 2014. Annual report of the European antimicrobial resistance surveillance network (EARS-Net), ECDC, Stockholm, 2015.

2. Magill, S. S., Edwards, J. R., Bamberg, W., Beldavs, Z. G., Dumyati, G., Kainer, M. A., Lynfield, R., Maloney, M., McAllister-Hollod, L., Nadle, J., Ray, S., Thompson, D. L., Wilson, L. E., Fridkin, S. C.: Multistate point-prevalence survey of health care-associated infections. N Engl J Med 370, 1198-1208 (2014).

3. Bal, A. M., Coombs, G. W., Holden, M. T. G., Lindsay, J. A., Nimmo, G. R., Tattevin, P., Skov, R. L.: Genomic insights into the emergence and spread of international clones of healthcare-, community- and livestock-associated meticillin-resistant Staphylococcus aureus: Blurring of the traditional definitions. J Glob Antimicrob Resist 6, 95-101 (2016)

4. Otto, M.: Community-associated MRSA: What makes them special? Int J Med Microbiol IJMM 303, 324-330 (2013)

5. Stefani, S., Chung, D. R., Lindsay, J. A., Friedrich, A. W., Kearns, A. M., Westh, H., Mackenzie, F. M.: Meticillin-resistant Staphylococcus aureus (MRSA): Global epidemiology and harmonisation of typing methods. Int J Antimicrob Agents 39, 273-282 (2012).

6. Bush, K., Leal, J., Fathima, S., Li, V., Vickers, D., Chui, L., Louie, M., Taylor, G., Henderson, E.: The molecular epidemiology of incident methicillin-resistant Staphylococcus aureus cases among hospitalized patients in Alberta, Canada: A retrospective cohort study. Antimicrob Resist Infect Control 4, 35 (2015).

7. Aires-de-Sousa, M.: Methicillin-resistant Staphylococcus aureus among animals: Current overview. Clin Microbiol Infect 23, 373-380 (2017).

8. van Cleef, B. A. G. L., Monnet, D. L., Voss, A., Krziwanek, K., Allerberger, F., Struelens, M., Zemlickova, H., Skov, R. L., Vuopio-Varkila, J., Cuny, C., Friedrich, A. W., Spiliopoulou, I., 
Pászti, J., Hardardottir, H., Rossney, A., Pan, A., Pantosti, A., Borg, M., Grundmann, H., Mueller-Premru, M., Olsson-Liljequist, B., Widmer, A., Harbarth, S., Schweiger, A., Unal, S., Kluytmans, J. A.: Livestock-associated methicillin-resistant Staphylococcus aureus in humans, Europe. Emerg Infect Dis 17, 502-505 (2011).

9. Cuny, C., Wieler, L. H., Witte, W.: Livestock-associated MRSA: The impact on humans. Antibiot Basel Switz 4, 521-543 (2015).

10. Price, L. B., Stegger, M., Hasman, H., Aziz, M., Larsen, J., Andersen, P. S., Pearson, T., Waters, A. E., Foster, J. T., Schupp, J., Gillece, J., Driebe, E., Liu, C. M., Springer, B., Zdovc, I., Battisti, A., Franco, A., Zmudzki, J., Schwarz, S., Butaye, P., Jouy, E., Pomba, C., Porrero, M. C., Ruimy, R., Smith, T. C., Robinson, D. A., Weese, J. S., Arriola, C. S., Yu, F., Laurent, F., Keim, P., Skov, R., Aarestrup, F. M.: Staphylococcus aureus CC398: Host adaptation and emergence of methicillin resistance in livestock. mBio 3, e00305-11 (2012).

11. Kinross, P., Petersen, A., Skov, R., Van Hauwermeiren, E., Pantosti, A., Laurent, F., Voss, A., Kluytmans, J., Struelens, M. J., Heuer, O., Monnet, D. L., The European Human LA-Mrsa Study Group: Livestock-associated meticillin-resistant Staphylococcus aureus (MRSA) among human MRSA isolates, European Union/European Economic Area countries, 2013. Euro Surveill 22, 16-00696 (2017).

12. European Centre for Disease Prevention and Control: Point Prevalence Survey of Health Care Associated Infections and Antimicrobial Use in European Acute Care Hospitals. ECDC, Stockholm, 2013.

13. Kolman, A., Lejko Zupanc, T., Kotnik-Kevorkijan, B., Klavs, I., Korošec, A., Serdt, M.: Prevalenca proti antibiotikom odpornih povzročiteljev okužb v slovenskih bolnišnicah za akutno oskrbo [Prevalence of antibiotic-resistant pathogens of healthcare-associated infections in Slovenian hospitals for acute care]. Med Razgledi 52, 23-28 (2013).

14. Henderson, D. K.: Managing methicillin-resistant staphylococci: A paradigm for preventing nosocomial transmission of resistant organisms. Am J Med 119, S45-52; discussion S62-S70 (2006).

15. Müller-Premru, M., Strommenger, B., Alikadic, N., Witte, W., Friedrich, A. W., Seme, K., Kucina, N. S., Smrke, D., Spik, V., Gubina, M.: New strains of community-acquired methicillin-resistant Staphylococcus aureus with Panton-Valentine leukocidin causing an outbreak of severe soft tissue infection in a football team. Eur J Clin Microbiol Infect Dis 24, 848-850 (2005).

16. Dermota, U., Grmek-Košnik, I., Ravnik, M., Budimir, A., Ribič, H., Cerkvenik-Škafar, A.: First report of community-acquired meticillin-resistant Staphylococcus aureus from a Slovenian hospital. J Hosp Infect 79, 271-272 (2011).

17. Dermota, U., Jurca, T., Harlander, T., Košir, M., Zajc, U., Golob, M., Zdovc, I., Košnik, I. G.: Infections caused by community-associated methicillin-resistant Staphylococcus aureus European clone (ST80) in Slovenia between 2006 and 2013. Zdr Varst 55, 121-125 (2016).

18. Dermota, U., Zdovc, I., Strumbelj, I., Grmek-Kosnik, I., Ribic, H., Rupnik, M., Golob, M., Zajc, U., Bes, M., Laurent, F., Mueller-Premru, M.: Detection of methicillin-resistant Staphylococcus aureus carrying the mecC gene in human samples in Slovenia. Epidemiol Infect 143, 1105-1108 (2015). 
19. Dermota, U., Mueller-Premru, M., Švent-Kučina, N., Petrovič, Ž., Ribič, H., Rupnik, M., Janežič, S., Zdovc, I., Grmek-Košnik, I.: Survey of community-associated-methicillinresistant Staphylococcus aureus in Slovenia: Identification of community-associated and livestock-associated clones. Int J Med Microbiol IJMM 305, 505-510 (2015).

20. Grundmann, H., Schouls, L. M., Aanensen, D. M., Pluister, G. N., Tami, A., Chlebowicz, M., Glasner, C., Sabat, A. J., Weist, K., Heuer, O., Friedrich, A. W.: The dynamic changes of dominant clones of Staphylococcus aureus causing bloodstream infections in the European region: Results of a second structured survey. Euro Surveill Bull Eur Sur Mal Transm Eur Commun Dis Bull 19, 20987 (2014).

21. Hetem, D. J., Derde, L. P. G., Empel, J., Mroczkowska, A., Orczykowska-Kotyna, M., Kozińska, A., Hryniewicz, W., Goossens, H., Bonten, M. J. M., MOSAR WP3 Study Group: Molecular epidemiology of MRSA in 13 ICUs from eight European countries. J Antimicrob Chemother 71, 45-52 (2016).

22. Kotnik-Kevorkijan, B., Petrovič, Ž., Klasinc, M., Rupnik, M., Lorenčič Robnik, S.: Diversity of spa types among MRSA isolates from Maribor University Hospital. Zdr Vestn 78, 119-122 (2009).

23. Grisold, A. J., Leitner, E., Mühlbauer, G., Marth, E., Kessler, H. H.: Detection of methicillin-resistant Staphylococcus aureus and simultaneous confirmation by automated nucleic acid extraction and real-time PCR. J Clin Microbiol 40, 2392-2397 (2002).

24. Shrestha, N. K., Tuohy, M. J., Hall, G. S., Isada, C. M., Procop, G. W.: Rapid identification of Staphylococcus aureus and the mecA gene from BacT/ALERT blood culture bottles by using the LightCycler system. J Clin Microbiol 40, 2659-2661 (2002).

25. Harmsen, D., Claus, H., Witte, W., Rothgänger, J., Claus, H., Turnwald, D., Vogel, U.: Typing of methicillin-resistant Staphylococcus aureus in a university hospital setting by using novel software for spa repeat determination and database management. J Clin Microbiol 41, 5442-5448 (2003).

26. Mellmann, A., Weniger, T., Berssenbrügge, C., Rothgänger, J., Sammeth, M., Stoye, J., Harmsen, D.: Based Upon Repeat Pattern (BURP): An algorithm to characterize the long-term evolution of Staphylococcus aureus populations based on spa polymorphisms. BMC Microbiol 7, 98 (2007).

27. Monecke, S., Coombs, G., Shore, A. C., Coleman, D. C., Akpaka, P., Borg, M., Chow, H., Ip, M., Jatzwauk, L., Jonas, D., Kadlec, K., Kearns, A., Laurent, F., O’Brien, F. G., Pearson, J., Ruppelt, A., Schwarz, S., Scicluna, E., Slickers, P., Tan, H. L., Weber, S., Ehricht, R.: A field guide to pandemic, epidemic and sporadic clones of methicillin-resistant Staphylococcus aureus. PLoS One 6, e17936 (2011).

28. Asadollahi, P., Farahani, N. N., Mirzaii, M., Khoramrooz, S. S., van Belkum, A., Asadollahi, K., Dadashi, M., Darban-Sarokhalil, D.: Distribution of the most prevalent spa types among clinical isolates of methicillin-resistant and -susceptible Staphylococcus aureus around the world: A review. Front Microbiol 9, 163 (2018).

29. Strommenger, B., Kettlitz, C., Weniger, T., Harmsen, D., Friedrich, A. W., Witte, W.: Assignment of Staphylococcus isolates to groups by spa typing, SmaI macrorestriction analysis, and multilocus sequence typing. J Clin Microbiol 44, 2533-2540 (2006).

30. Cirkovic, I., Stepanovic, S., Skov, R., Trajkovic, J., Grgurevic, A., Larsen, A. R.: Carriage and genetic diversity of methicillin-resistant Staphylococcus aureus among patients and healthcare workers in a Serbian university hospital. PLoS One 10, e0127347 (2015) 
31. Ostojić, M., Hukić, M.: Genotypic and phenotypic characteristics of methicillin-resistant Staphylococcus aureus (MRSA) strains, isolated on three different geography locations. Bosn J Basic Med Sci 15, 48-56 (2015)

32. Cuny, C., Layer, F., Werner, G., Harmsen, D., Daniels-Haardt, I., Jurke, A., Mellmann, A., Witte, W., Köck, R.: State-wide surveillance of antibiotic resistance patterns and spa types of methicillin-resistant Staphylococcus aureus from blood cultures in North RhineWestphalia, 2011-2013. Clin Microbiol Infect 21, 750-757 (2015).

33. Nillius, D., von Müller, L., Wagenpfeil, S., Klein, R., Herrmann, M.: Methicillin-resistant Staphylococcus aureus in Saarland, Germany: The long-term care facility study. PLoS One 11, e0153030 (2016)

34. Brennan, G. I., Abbott, Y., Burns, A., Leonard, F., McManus, B. A., O'Connell, B., Coleman, D. C., Shore, A. C.: The emergence and spread of multiple livestock-associated clonal complex 398 methicillin-resistant and methicillin-susceptible Staphylococcus aureus strains among animals and humans in the Republic of Ireland, 2010-2014. PLoS One 11, e0149396 (2016).

35. Zarfel, G., Luxner, J., Folli, B., Leitner, E., Feierl, G., Kittinger, C., Grisold, A.: Increase of genetic diversity and clonal replacement of epidemic methicillin-resistant Staphylococcus aureus strains in South-East Austria. FEMS Microbiol Lett 363, fnw137 (2016).

36. Cuny, C., Köck, R., Witte, W.: Livestock associated MRSA (LA-MRSA) and its relevance for humans in Germany. Int J Med Microbiol IJMM 303, 331-337 (2013).

37. Becker, K., Ballhausen, B., Kahl, B. C., Köck, R.: The clinical impact of livestock-associated methicillin-resistant Staphylococcus aureus of the clonal complex 398 for humans. Vet Microbiol 200, 33-38 (2015). 